\title{
Evaluación del espesor de capas delgadas mediante análisis EDS y simulación de Monte Carlo
}

\author{
J.C. García* y F. Peñalba
}

\begin{abstract}
Resumen
En este trabajo se presenta un método de evaluación de espesores de capas delgadas mediante la técnica de energías dispersivas de rayos X (EDS). Las medidas analíticas de las áreas de los picos característicos de los elementos detectados en las capas, obtenidas mediante esta técnica, se complementan con simulaciones de Monte Carlo de las trayectorias de los electrones en el volumen de interacción. Esto, junto con las estimaciones de la intensidad de los picos característicos obtenidos mediante el algoritmo de Bethe, Joy \& Luo, posibilita realizar la estimación de los espesores de las muestras analizadas.
\end{abstract}

Palabras clave Capas finas nanométricas; Monte Carlo; EDS; Aceros; Materiales metálicos..

\section{Thin layer thickness evaluation by EDS analysis and Monte Carlo simulation}

\begin{abstract}
This work shows a method to evaluate the thin layer thickness by the $\mathrm{X}$ ray dispersive energy (EDS) technique. The analytical measures of the area of characteristic peaks carried out with this technique are complemented with electron trajectories on the interaction volume by Monte Carlo simulation. These together with the estimations obtained for the characteristic peaks intensities according to Bethe, Joy \& Luo algorithm makes possible to estimate the thickness of samples.
\end{abstract}

Keywords

Nanometric thin layers; Monte Carlo; EDS: Steels; Metallic materials..

\section{INTRODUCCIÓN}

Actualmente, la industria de fabricación de chapa y de productos planos utiliza recubrimientos cada vez más finos para la protección de las chapas laminadas. También, hay casos en los que es necesario evaluar el espesor de los óxidos que se forman en superficie. Existen técnicas apropiadas para la medida de las capas finas, tales como Auger, XPS, SIMS, FIBTEM; sin embargo, este tipo de equipamiento es caro y su disponibilidad escasa y, por otra parte, el ensayo en sí mismo suele ser lento.

Sin llegar a ser equipos de uso común, la disponibilidad de equipos de microscopía electrónica con microsonda EDS suele ser una opción más común en grandes empresas, centros de investigación o universidades. Sin embargo, esta técnica cuenta con el hándicap de que su profundidad característica de análisis suele ser del orden de las unidades de micra por lo que, en caso de espesores menores, las señales obte- nidas proceden no solo de la capa sino también del substrato. Además, se trata de una técnica eminentemente analítica y no de medida de espesores.

Se presenta, en este trabajo, un método de cálculo numérico que permite, mediante evaluación de las intensidades de los picos característicos, la estimación del espesor de capas finas por debajo de la micra. El método se basa en dos estimaciones. Una primera estimación para la determinación de las trayectorias de los electrones en el material, en el volumen de interacción entre ambos utilizando la técnica de Monte Carlo. Para ello, se suponen capas de espesor conocido sobre el sustrato del material. La segunda aproximación se realiza mediante el cálculo de las intensidades teóricas de los picos característicos de los elementos presentes en los casos supuestos. Con todo ello, se consigue determinar unas curvas de calibración relacionando las intensidades de pico frente al espesor de la capa. 


\section{MÉTODO EXPERIMENTAL}

Los análisis se realizaron mediante una microsonda OXFORD-INCA300 acoplada a un microscopio electrónico de barrido JEOL JSM-5910 LV. Se contrastaron los resultados obtenidos mediante esta técnica con los valores obtenidos mediante un espectrómetro de superficies XPS de Microlab MkII de la casa VG Scientific.

La simulación se realizó tomando el algoritmo de Bethe, Joy \& Luo, que posibilita la evaluación de la áreas características de los picos de los elementos detectados por la técnica EDS.

Las muestras que se han analizado corresponden a dos tipos diferentes: uno de ellos, son chapas de acero electrozincadas recubiertas mediante silanos, por vía líquida (silano) y, otro tipo, corresponde a las mismas chapas de acero pero el recubrimiento de silanos se realizó mediante CVD (CVD). En cada tipo de muestra se obtuvieron tres espesores de recubrimiento, denominados fino, medio y grueso, en función del tiempo de aplicación del recubrimiento. La chapa inicial, previa al recubrimiento, presentaba un acabado de rugosidad media Ra: 1,15 $\mu \mathrm{m}$.

\section{RESULTADOS Y DISCUSIÓN}

La figura 1 muestra el aspecto superficial de una de las chapas analizadas. Se trata de una chapa recubierta con una capa de silano depositada mediante vía líquida y de espesor medio.

Se ha considerado, en esta simulación, que la estequiometría del silano de la capa es del tipo $\mathrm{H}_{4} \mathrm{Si}$ y se

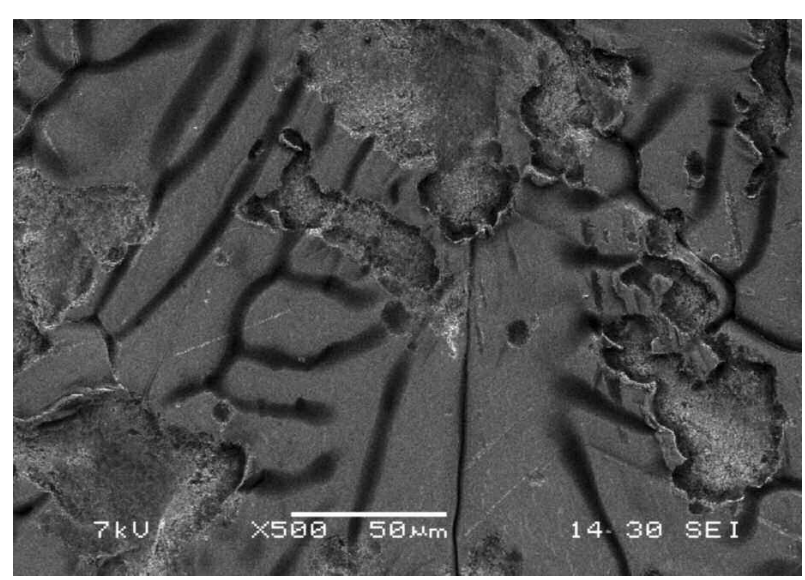

Figura 1. Aspecto superficial del recubrimiento silano en la capa media.

Figure 1. Silane medium coating, surface aspect. han simulado espesores de capa de 5, 10, 30, 50, 100, 300,500 y $1.000 \mathrm{~nm}$.

La figura 2 muestra la simulación para un número significativo de 2.000 trayectorias. Se puede observar el volumen de interacción de los electrones con la muestra. Una vez obtenidas las trayectorias, es posible determinar el área de los picos característicos obtenidos mediante EDS para altas energías, con ayuda de las relaciones de Bethe ${ }^{[1]}$ para el volumen de interacción y la intensidad de los rayos-X característicos. Para bajas energías se aplica la relación de Bethe y Powell[2]. La simulación, en estas condiciones, proporciona las siguientes intensidades (Fig. 3): para los picos característicos del silicio, intensidad de silicio $=12$ (representativo del recubrimiento de silano) $\mathrm{y}$ zinc, intensidad de zinc $=1.116$ (representativo del sustrato, chapa electrozincada $)^{[3]}$.

Debido a que en esta simulación se consideraron 2.000 trayectorias de electrones, pero no se conoce el número de electrones emitidos en el caso real, se determinó la relación entre las intensidades de los picos silicio y zinc, Si/Zn. Con ello, se normalizan las relaciones de intensidad de los picos para que no se vea afectada la intensidad respecto al número de electrones emitidos. Posteriormente, se determinó la misma relación para otros espesores de capa. Así, se puede representar la relación de intensidades frente al espesor de capa (Fig. 4).

De esta curva es posible obtener la curva de regresión que mejor se ajusta a estos datos. Se obtiene una ecuación de ajuste:

$$
y=837,39 x^{0,8493}
$$

con un coeficiente de correlación, $\mathrm{R}^{2}=0,997$.

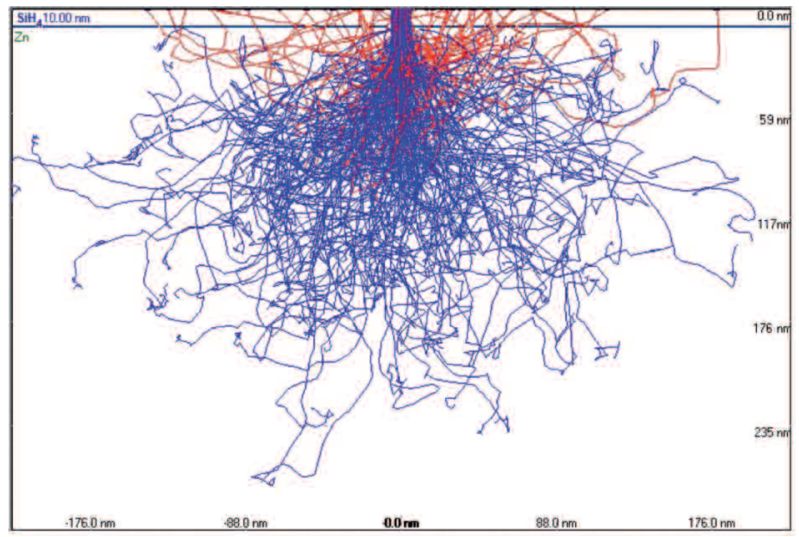

Figura 2. Simulación de Monte Carlo obtenida sobre una capa de $10 \mathrm{~nm}$ de recubrimiento.

Figure 2. Monte Carlo simulation on a $10 \mathrm{~nm}$ coating layer thickness. 

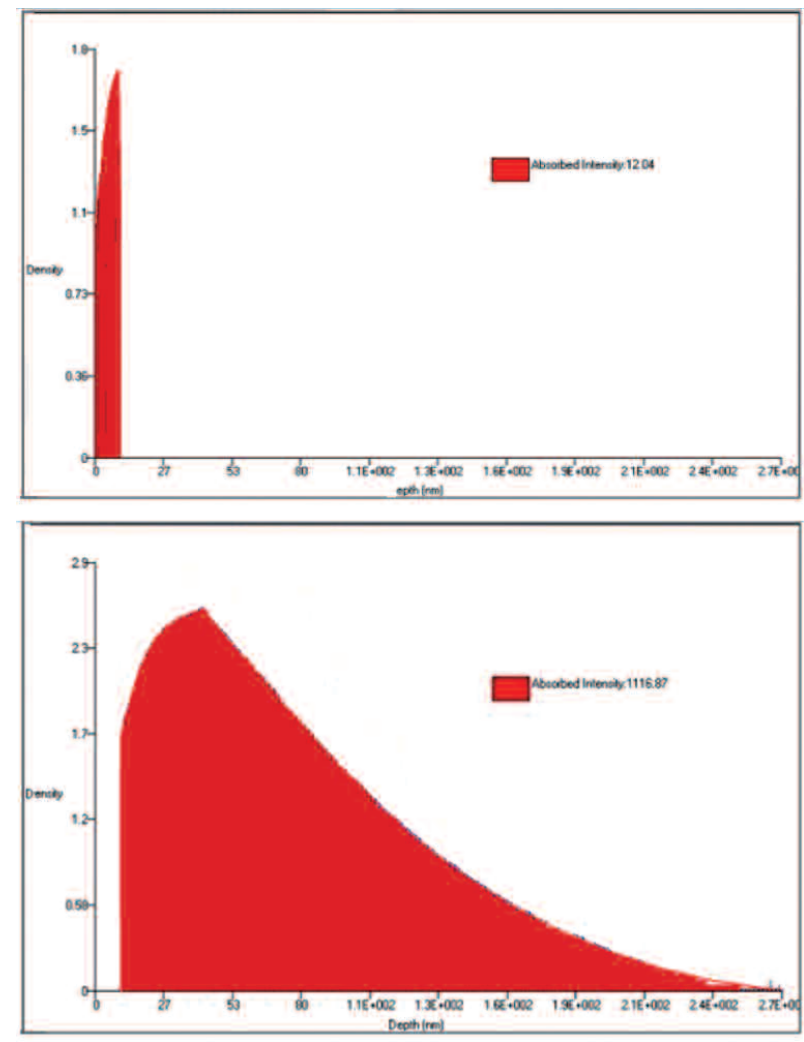

Figura 3. Intensidades obtenidas para los picos de silicio (12) y zinc (1.116), suponiendo un recubrimiento de $10 \mathrm{~nm}$.

Figure 3. Intensities for silicon (12) and zinc $(1,116)$ peaks on a $10 \mathrm{~nm}$ thickness coating.

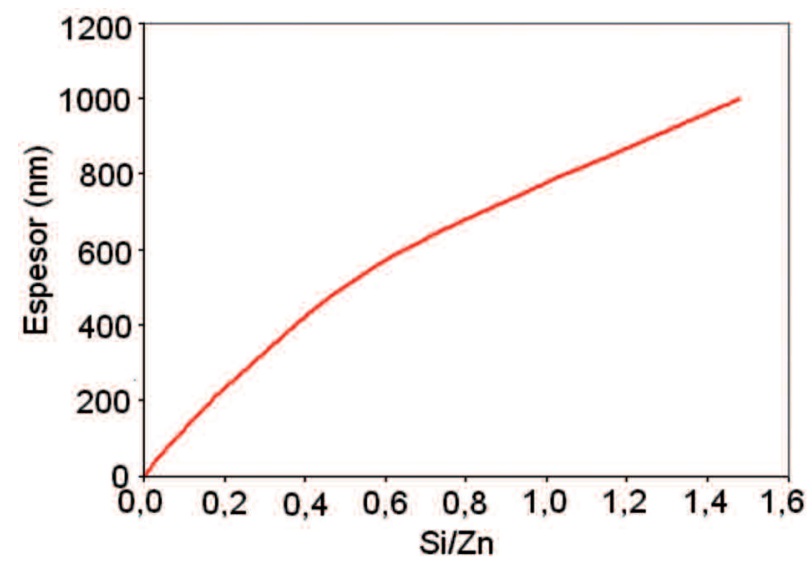

Figura 4. Relación de intensidades Si/Zn frente a espesor de capa.

Figure 4. Si/Zn intensities rate vs. layer thickness.

Una vez obtenida la curva de calibración se puede evaluar la misma relación de elementos en las muestras estudiadas y de ahí deducir el espesor.
Sobre las muestras de chapa recubierta con silano por vía líquida (silano) y de espesor medio (Fig. 5), se muestra el espectro EDS obtenido sobre el recubrimiento. Las intensidades medidas para el silicio y zinc son, respectivamente, 1.995 y 4.521.

En este caso, la relación $\mathrm{Si} / \mathrm{Zn}$ resulta ser 0,4414 y, de ahí, se obtiene un espesor para este recubrimiento estimado de $418 \mathrm{~nm}$. Las muestras de recubrimiento de silano depositados mediante CVD (CVD) se evalúan de la misma manera a partir de la ecuación de ajuste determinada ya que en la simulación de Monte Carlo y la estimación de las áreas de los picos, no se ha limitado a ningún tipo de proceso de recubrimiento.

Repitiendo el mismo proceso para el resto de las muestras se obtienen los siguientes resultados que se muestran en la tabla I.

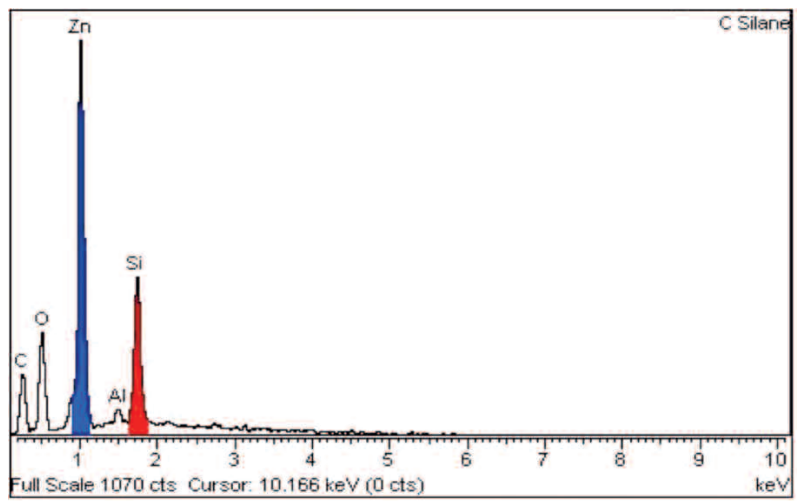

Figura 5. Espectro EDS obtenido sobre la muestra de recubrimiento de silano por vía líquida y de espesor medio.

Figure 5. EDS spectrum obtained on a silane medium size coating.

Tabla I. Relación de intensidades de los picos característicos seleccionados y espesor estimado

Table I. Intensities rate from characteristic selected peaks according to simulated thickness

\begin{tabular}{lccc}
\hline \multicolumn{2}{c}{ Recubrimiento } & Si/Zn & Espesor $(\mathbf{n m})$ \\
\hline \multirow{4}{*}{ Silano } & Fino & 0,1986 & 212 \\
& Medio & 0,4414 & 418 \\
& Grueso & 1,3102 & 1.053 \\
CVD & Fino & 0,1602 & 177 \\
& Medio & 0,3363 & 332 \\
& Grueso & 0,7541 & 659 \\
\hline
\end{tabular}


Para confirmar la bondad del sistema de determinación de los espesores mediante la relación de los picos característicos de silicio y zinc, se determinaron los espesores del recubrimiento de las capas de silano, realizándose medidas de decapado iónico mediante la técnica XPS. Los resultados se muestran en la figura 6. En esta, se muestra el espectro obtenido sobre el primer nivel de decapado de la muestra recubierta con silano vía líquida y de espesor medio. Se aprecian los picos característicos de las transiciones electrónicas de los orbitales del oxígeno, carbono, silicio y con mucha menor intensidad del zinc.

Con objeto de determinar el espesor de la capa de recubrimiento mediante esta técnica de análisis $\mathrm{XPS}^{[4]}$, se realizó un decapado iónico mediante iones de argón acelerados de forma iterativa, que permiten eliminar las capas superficiales del material al mismo tiempo que se realizan análisis químicos que indican los elementos que permanecen en la superficie de la muestra (un análisis y un cierto tiempo de "bombardeo" iónico de forma repetitiva), tras el bombardeo iónico. Posteriormente, una calibración del tiempo de decapado permite convertir los datos de este tiempo a espesor de material eliminado.

A partir del espectro XPS inicial, se procedió a decapar la superficie de la muestra a intervalos de 10 min, al cabo de los cuales se realizó un nuevo análisis representándose los resultados de elementos de interés de la composición obtenida frente al tiempo. La figura 7 muestra el perfil de evolución de los elementos obtenidos mediante XPS frente al tiempo de decapado. Se aprecia que, tras un análisis inicial en el que el carbono alcanza un $20 \%$, se produce un decrecimiento de este, por lo que la presencia del mismo en la primera capa puede ser achacada a la

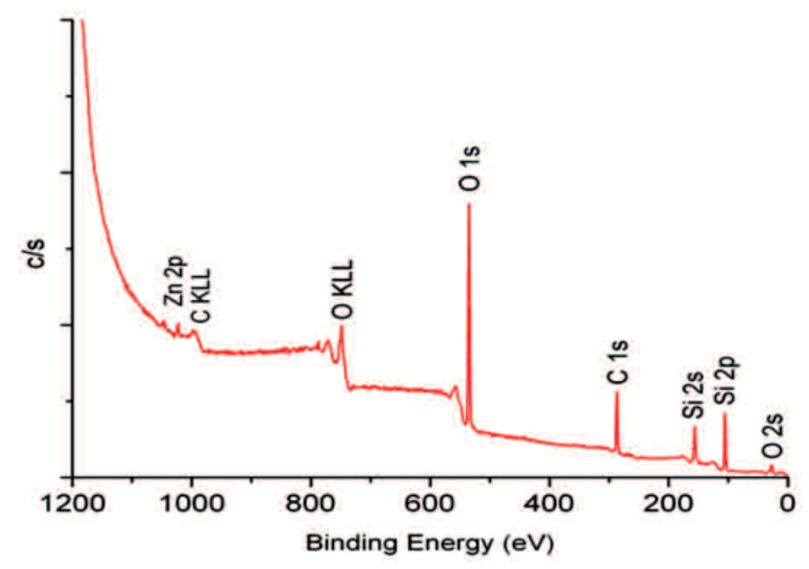

Figura 6. Espectro XPS obtenido sobre la muestra de recubrimiento medio en silano.

Figure 6. XPS spectrum obtained on a silane medium coating.

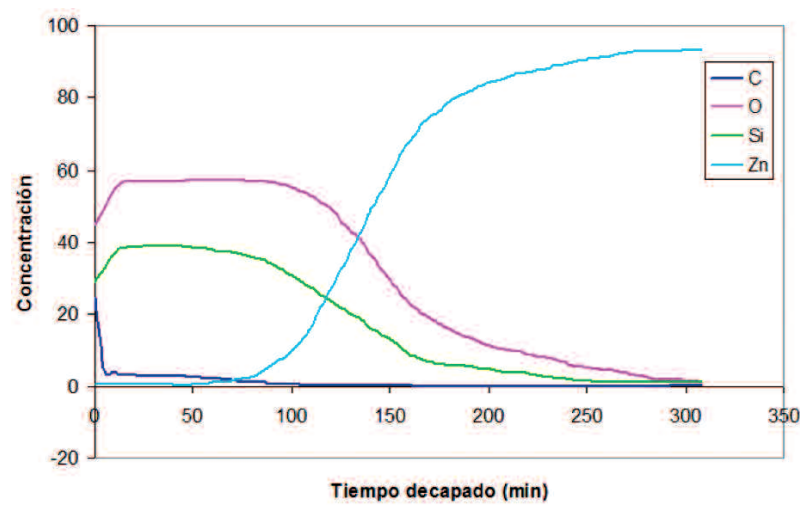

Figura 7. Perfil en profundidad obtenido mediante decapado iónico y análisis XPS a una muestra de silano con recubrimiento medio.

Figure 7. Depth profile after ionic etching and XPS analysis on a medium size silane coating.

contaminación exterior. A continuación, se aprecia un intervalo, hasta aproximadamente $100 \mathrm{~min}$, de decapado en el que silicio, oxígeno y zinc presentan una concentración estable y que sería la correspondiente a la capa de silano sobre el sustrato de zinc. A partir de este punto, la concentración de zinc aumenta gradualmente mientras que las de oxígeno y silicio decrecen; esta zona correspondería a la zona de difusión existente entre la capa y sustrato. En todo caso, no se observa un punto exacto de desaparición del silicio (como elemento característico de la capa) ni una aparición abrupta del zinc (como elemento que permita definir el sustrato).

En los casos donde no es posible establecer un límite claro de aparición de un elemento o desaparición de otro, se establece como criterio de espesor de capa de silano, el tiempo necesario para alcanzar una concentración del $80 \%$ en zinc en el análisis químico de la capa correspondiente. En este caso, es de $182 \mathrm{~min}$, tal y como se observa en la misma figura 7.

Un problema común en esta técnica, a la hora de transformar los datos de tiempo de decapado en espesor real, es la dificultad de encontrar patrones convenientes para la transformación del tiempo de decapado iónico en espesor. Únicamente, existen algunos patrones comerciales de espesor definido para ciertas formulaciones muy concretas. Como patrón de transformación, se utilizó, en este trabajo, pentóxido de tantalio, $\mathrm{Ta}_{2} \mathrm{O}_{5}$, certificado por el National Institute of Standards and Technology (NIST).

Sin embargo, no existe una relación formal que permita establecer una equivalencia entre la velocidad de decapado para la muestra patrón antes indicada y la de los silanos presentes en el recubrimiento de las chapas estudiadas. Evidentemente, estos 
dos materiales, muestra patrón y muestra analizada, no presentarán la misma eficiencia al bombardeo iónico ya que los componen elementos tan dispares en número atómico como el tántalo y el silicio.

Por todo ello, los valores aquí obtenidos y resumidos en la tabla II, tampoco han de ser considerados como totalmente exactos.

Precisamente, uno de los objetivos del proyecto europeo en el que se desarrolló este trabajo, fue definir la técnica y el método mejor para evaluar los espesores de estas capas nanométricas. Por ello, se dispuso también de la posibilidad de realizar una serie de medidas mediante la técnica FIB-TEM [5] (Fig. 8). Sobre una reducida selección de muestras

Tabla II. Tiempo necesario en alcanzar el $80 \%$ de zinc y espesor de capa equivalente.

Table II. Time needed to reach an $80 \%$ of zinc and equivalent layer

\begin{tabular}{lccc}
\hline Capa & $\begin{array}{c}\text { Tiempo } \\
(\mathbf{m i n})\end{array}$ & $\begin{array}{c}\text { Espesor } \\
(\mathbf{n m})\end{array}$ \\
\hline \multirow{4}{*}{ Silano } & Fino & 55 & 174 \\
& Medio & 182 & 584 \\
& Grueso & 273 & 876 \\
CVD & Fino & 47 & 152 \\
& Medio & 92 & 295 \\
& Grueso & 184 & 589 \\
\hline
\end{tabular}

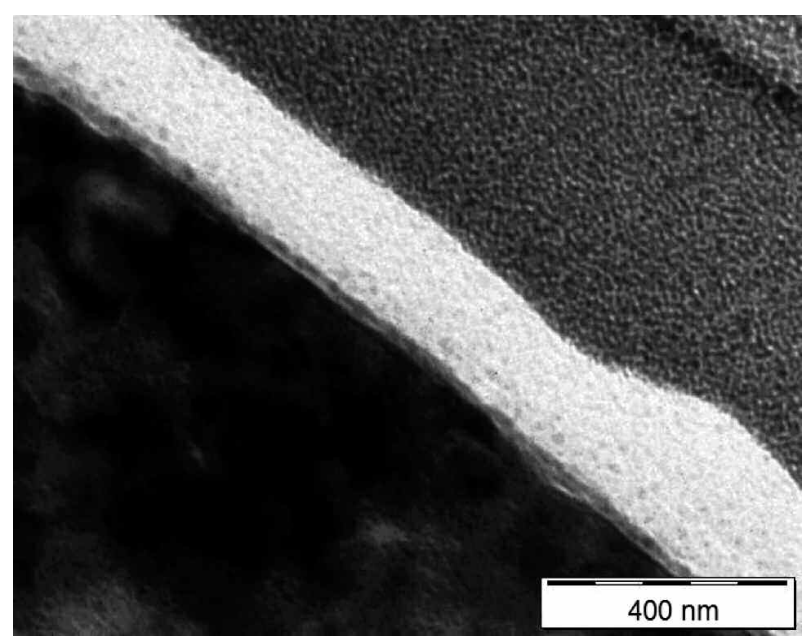

Figura 8. Imagen obtenida mediante FIB-TEM de la muestra recubierta por CVD en espesor medio.

Figure 8. FIB-TEM image of a sample CVD medium coated. para su observación, se obtuvieron unas imágenes mediante la técnica indicada.

Sobre las imágenes obtenidas mediante esta técnica, ha sido posible medir el espesor de las capas depositadas. Se ha determinado que existen notables variaciones en la medida de los recubrimientos según el proceso de recubrimiento aplicado. Así, ha resultado que el recubrimiento denominado fino en silano oscila entre 130 y $640 \mathrm{~nm}$, para el caso del recubrimiento realizado mediante inmersión en vía líquida (silano). Sin embargo, la medida del espesor del recubrimiento de silano realizado mediante CVD (CVD), de espesor medio, presenta menores dispersiones que en los anteriores. Los valores varían, sólo, entre 160 y $240 \mathrm{~nm}$.

La gran diferencia que se obtiene entre estos espesores de silano depositado por las dos técnicas (los espesores encontrados en las muestras CVD, de espesor medio, están incluidos en la muestras de silano de espesor fino) se debe al proceso de fabricación seguido en cada técnica de deposición ${ }^{[6]}$. En el denominado silano, depositado por vía líquida, se consigue la capa mediante un proceso de inmersión en fase líquida. Este proceso tiende a recubrir y homogeneizar todos los defectos superficiales que pudiera presentar la chapa previamente a su recubrimiento; en este caso, queda reducida las diferencias existentes entre "valles" y "picos" de la propia rugosidad o de otros defectos superficiales. Por el contrario, el proceso de recubrimiento por CVD se realiza en fase sólida, por lo que la capa replica la superficie de la muestra, manteniendo la capa la rugosidad de la chapa (Fig. 9).
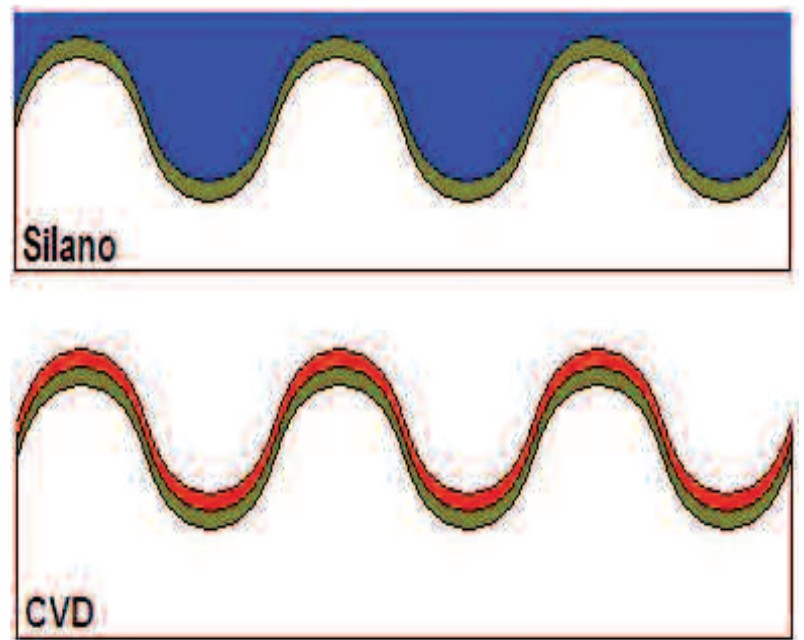

Figura 9. Diferencia en el tipo de recubrimiento según proceso.

Figure 9. Thickness difference according to process coating. 


\section{CONCLUSIONES}

Se ha presentado un método de evaluación de espesores de capas nanométricas, mediante las medidas analíticas de las áreas de los picos característicos de los elementos detectados empleando las técnicas de energías dispersivas de rayos X (EDS) asociadas a la microscopía electrónica de barrido (MEB), complementada con simulaciones de Monte Carlo, en los casos en los que no se dispone de equipamiento específico para tales medidas.

Si bien es cierto que la aplicación de las técnicas de Monte Carlo en zonas amplias se alejan de las condiciones ideales, los valores obtenidos para la estimación del espesor sobre este tipo de recubrimientos y sustratos no presenta discrepancias frente a medidas realizadas con otras técnicas más costosas.

Medidas realizadas mediante esta metodología deberán, siempre, ser contrastadas, inicialmente, con otras técnicas para evaluar su aplicación, tanto en función de los materiales presentes en el recubrimiento y sustrato, así como frente al rango de medidas del espesor obtenido. En las técnicas de medida de las capas a utilizar ha de tenerse en cuenta el proceso de recubrimiento utilizado ya que puede inducir a errores en la medida real de la capa.

El rango de precisión de los espesores obtenidos mediante esta técnica, considerando que su espesor real de los silanos es desconocido en las muestras estudiadas, se puede considerar satisfactorio. Así, de los valores obtenidos por la técnica antes descrita frente a los valores obtenidos mediante la técnica XPS con decapado, el mayor error supone un $28 \%$, correspondiente a la muestra de silano de espesor medio recubierta vía líquida.

La discrepancia encontrada en las medidas realizadas por las dos técnicas, en las muestras de silano recubiertas vía líquida, varía entre un 20 y un $28 \%$, mientras que la discrepancia para los espesores obte- nidos sobre silano recubierto vía CVD oscila entre un 11 y un $16 \%$, menor que el caso anterior, debido al proceso de recubrimiento.

A la vista de los resultados, una vez validada la metodología propuesta en este trabajo mediante la aplicación de algunas de las técnicas más específicas, para un caso concreto, esta sería de utilidad en los casos en los que se requiere un gran número de medidas de espesores, siempre que no cambie la naturaleza de los materiales aplicados.

\section{Agradecimientos}

Este trabajo ha sido financiado por el Proyecto de la Comunidad Europea RFS-CR-04171.

\section{REFERENCIAS}

[1] D.C. Joy, A.D. Romig y J.I. Goldstein, Principles of analytical electron microscopy, Plenum Press, Nueva York, EE. UU., 1989, pp. 3-9.

[2] D.E. Newbury, D.C. Joy, P. Exhlin, C.E. Fiori y J.I. Goldstein, Advanced scanning electron microscopy and X-ray microanalysis, Plenum Press, Nueva York, EE. UU., 1987, pp. 7-19.

[3] J.M. Palacios, J.L. Arana, J.I. Larburu y L. Iniesta, La fabricación del acero, Ed. Spainfo, Unesid, Madrid, España, 2002.

[4] J.M. Walls, Methods of surface analysis: techniques and applications. Cambridge University Press, Inglaterra, 1989, pp. 127-168.

[5] L.A. Giannuzzi y F.A. Stevie, Introduction to focused ion beams, Springer Science, Nueva York, EE. UU., 2005, pp. 1-12.

[6] Materials science and technology: a comprehensive treatment. Vol. 7. Ed. R.W. Cahn P. Haasen y E.J. Kramer, VCH, Nueva York, EE. UU., 1992, pp. 792-797. 